\title{
Al(111)- $(\sqrt{3} \times \sqrt{3})$ R30: On-top versus substitutional adsorption for $\mathbf{R b}$ and $\mathbf{K}$
}

\author{
P. Scharoch, * J. Neugebauer, and M. Scheffler \\ Fritz-Haber-Institut der Max-Planck-Gesellschaft, Faradayweg 4-6, D-14195 Berlin, Germany
}

(Received 4 February 2003; published 1 July 2003)

\begin{abstract}
The atomic structure and energetics of the on-top and substitutional phase of the $\operatorname{Al}(111)-(\sqrt{3} \times \sqrt{3}) R 30: \mathrm{Rb}$ adsorbate system have been studied employing density-functional-theory total-energy calculations. Since the energy difference between the two phases is extremely small $(\approx 20 \mathrm{meV})$ extensive checks with respect to the choice of the exchange-correlation functional (local density and generalized gradient approximations) and the construction of the Rb pseudopotential have been performed. All tests clearly show the substitutional adsorption site to be energetically favorable. Equivalent calculations performed for $\mathrm{K}$ revealed a clear chemical trend in the adsorption energetics for $\mathrm{Na}, \mathrm{K}$, and $\mathrm{Rb}$ : The energy gain between the substitutional and the on-top configuration decreases with increasing atomic radius of an alkali atom. The calculated equilibrium geometries are in good agreement with previously reported experimental data.
\end{abstract}

DOI: 10.1103/PhysRevB.68.035403

PACS number(s): 68.43. $-\mathrm{h}, 68.43 . \mathrm{Bc}$

\section{INTRODUCTION}

Over the past 15 years extensive experimental and theoretical investigations of alkali adsorbates on metal surfaces have revealed a great variety of ordered adsorbate structures and surface alloys. ${ }^{1-12}$ Among these structures the ordered $\mathrm{Al}(111)-(\sqrt{3} \times \sqrt{3}) R 30: X$ surface (with $1 / 3$ monolayer of $X$ $=\mathrm{Na}, \mathrm{K}, \mathrm{Rb}, \mathrm{Cs})$ probably has one of the simplest structures. Nevertheless, the system exhibits interesting structural phase transitions. ${ }^{4-6,10,11,13}$ Rubidium and potassium, e.g., occupy on-top sites on the rumpled $\mathrm{Al}(111)$ surface if adsorbed at low temperatures $(T \approx 100 \mathrm{~K})$. However, when the structure is formed at room temperature the alkali atoms occupy sixfold-coordinated substitutional sites formed by kicking out every third surface Al atom. The same structure is achieved by adsorbing the alkali adsorbates at low temperatures and a subsequent anneal: An irreversible but orderpreserving transformation from the on-top structure to the substitutional phase occurs. This transition can be followed in temperature-dependent low-energy electron diffraction (LEED) studies. ${ }^{13}$ These studies allowed one to directly measure the transition temperature $T_{\text {act }}$ which was found to be $250 \mathrm{~K}$ for $\mathrm{Rb}$ (Ref. 11) and $210-220 \mathrm{~K}$ for $\mathrm{K}^{2,10}$ In the case of $\mathrm{Na}$ on $\mathrm{Al}(111)$ a slightly different behavior is observed. There is no experimental evidence that at low temperature the discussed on-top structure is formed and, as calculations show, ${ }^{3}$ in this case $\mathrm{Na}$ prefers the threefold-coordinated fcc site rather than the on-top configuration. However, at room temperature also the substitutional $\operatorname{Al}(111)-(\sqrt{3}$ $\times \sqrt{3}) R 30$ : Na phase occurs.

First-principles calculations have had a major impact in identifying and understanding these phenomena. ${ }^{1-3,6,9,14-16}$ For example, these calculations clearly showed that $1 / 3$ monolayer of $\mathrm{Na}$ on $\mathrm{Al}(111)$ prefers the substitutional site: At zero temperature the energy difference between substitutional and on-surface adsorption is $300 \mathrm{meV}$. For $\mathrm{K}$ the calculated adsorption energies for the substitutional and onsurface phase are almost degenerate, ${ }^{2,6}$ indicating that the chemical nature of the adsorbate strongly affects the bond- ing. For $\mathrm{Rb}$ on $\mathrm{Al}(111)$ first-principles calculations have not been reported so far.

In order to gain a better understanding of chemical trends for alkali adsorption on metal surfaces we have therefore studied the equilibrium geometry and energetics of the reconstructed $\mathrm{Al}(111)-(\sqrt{3} \times \sqrt{3}) R 30: \mathrm{Rb}$ surface employing density-functional-theory calculations. Like in previous studies on $\mathrm{K}$ on $\mathrm{Al}(111)$ we find that the energy differences between substitutional and on-surface adsorption are almost degenerate. We have therefore performed extensive checks with respect to the choice of the pseudopotential, exchangecorrelation functional, size of the supercell, etc. An interesting conclusion based on these tests is that, in contrast to the early alkali adsorption models by Taylor and Langmuir ${ }^{17}$ and Gurney, ${ }^{18}$ semicore states significantly affect the chemical bonds. Polarization of the semicore states significantly reduces the energy difference between substitutional and ontop sites for both $\mathrm{K}$ and $\mathrm{Rb}$. It also significantly affects the bond length and cohesive energy of the elementary alkali bulk phases.

\section{METHOD}

\section{A. Ab initio calculations}

In order to calculate the equilibrium geometry and the adsorption energy of alkali adsorbates we have employed density functional theory using the FHI98MD plane-wave $\operatorname{code}^{19}$ and the FHIPP code ${ }^{20}$ to generate Troullier-Martins ${ }^{21}$ pseudopotentials. The surface has been described by a repeated slab geometry with the slab consisting of 4 layers of $\mathrm{Al}, 1 / 3$ layer of alkali atoms, and a vacuum thickness of $\approx 15 \AA$. The surface unit cell has a $(\sqrt{3} \times \sqrt{3}) R 30$ symmetry; i.e., each layer consists of three $\mathrm{Al}$ atoms. To obtain the equilibrium geometry the alkali adsorbates and the $\mathrm{Al}$ atoms in the first layer have been allowed to relax. The Brillouin zone has been sampled by a $(10,10,10)$ (for the elementary bulk phases of $\mathrm{Al}, \mathrm{K}, \mathrm{Rb}$ ) and by a $(6,6,1)$ (for the surfaces) Monkhorst-Pack mesh. The single-atom total energies, needed in the adsorbtion energy and cohesive energy calcu- 
TABLE I. Results of $a b$ initio calculations for bulk crystals of $\mathrm{Al}, \mathrm{Rb}$, and $\mathrm{K}$. Here $a_{\text {lat }}$ is the lattice constant, $B_{0}$ the bulk modulus, $E_{\text {coh }}$ the cohesive energy, $\delta(x)$ the deviation from the experimental value, and $E_{\text {cut }}$ the cutoff energy of the plane-wave basis set.

\begin{tabular}{cccccccccc}
\hline \hline \multirow{2}{*}{ Element } & $\begin{array}{c}\text { Data } \\
\text { from }\end{array}$ & $\begin{array}{c}\text { Valence } \\
\text { shell }\end{array}$ & $\begin{array}{c}E_{\text {cut }} \\
(\mathrm{Ry})\end{array}$ & $\begin{array}{c}a_{\text {lat }} \\
(\AA)\end{array}$ & $\begin{array}{c}\delta\left(a_{\text {lat }}\right) \\
(\%)\end{array}$ & $\begin{array}{c}B_{0} \\
(\mathrm{kbar})\end{array}$ & $\begin{array}{c}\delta\left(B_{0}\right) \\
(\%)\end{array}$ & $\begin{array}{c}E_{\text {coh }} \\
(\mathrm{eV})\end{array}$ & $\begin{array}{c}\delta\left(E_{\text {coh }}\right) \\
(\%)\end{array}$ \\
\hline \multirow{2}{*}{$\mathrm{Al}$} & Experiment & - & - & 4.049 & - & 760 & - & -3.38 & - \\
& LDA $^{\mathrm{a}}$ & $3 s^{2} 3 p^{1}$ & 20 & 3.971 & -1.9 & 850 & 12 & 4.04 & 19 \\
& LDA $^{\mathrm{a}}$ & $3 s^{2} 3 p^{1}$ & 45 & 3.971 & -1.9 & 840 & 11 & 4.04 & 19 \\
& GGA $^{\mathrm{b}}$ & $3 s^{2} 3 p^{1}$ & 30 & 4.022 & -0.7 & 756 & -1 & 3.67 & 8.5 \\
& GGA $^{\mathrm{b}}$ & $3 s^{2} 3 p^{1}$ & 50 & 4.024 & -0.6 & 761 & 0 & 3.58 & 6 \\
\hline \multirow{2}{*}{$\mathrm{K}$} & Experiment & - & - & 5.328 & - & 31 & - & 0.92 & - \\
& LDA $^{\mathrm{a}}$ & $4 s^{1}$ & 20 & 5.057 & -5.1 & 44 & 42 & 1.00 & 9 \\
& LDA $^{\mathrm{a}}$ & $3 p^{6} 4 s^{1}$ & 45 & 5.302 & -0.5 & 37 & 19 & 0.85 & -8 \\
& LDA $^{\mathrm{a}}$ & $3 p^{6} 4 s^{1}$ & 75 & 5.300 & -0.5 & 38 & 22 & 0.9 & -2 \\
& GGA $^{\mathrm{b}}$ & $3 p^{6} 4 s^{1}$ & 50 & 5.529 & 3.7 & 30 & -3 & 0.77 & -16 \\
& GGA $^{\mathrm{b}}$ & $3 p^{6} 4 s^{1}$ & 75 & 5.511 & 3.4 & 32 & 3 & 0.80 & -13 \\
\hline \multirow{2}{*}{$\mathrm{Rb}$} & Experiment & - & - & 5.585 & - & 25 & - & 0.84 & - \\
& LDA $^{\mathrm{a}}$ & $5 s^{1}$ & 20 & 5.355 & -4.1 & 36 & 44 & 0.94 & 12 \\
& LDA $^{\mathrm{a}}$ & $4 p^{6} 5 s^{1}$ & 25 & 5.571 & -0.2 & 31 & 24 & 0.80 & -5 \\
& LDA $^{\mathrm{a}}$ & $4 p^{6} 5 s^{1}$ & 60 & 5.571 & -0.2 & 30 & 20 & 0.82 & -2 \\
& GGA $^{\mathrm{b}}$ & $4 p^{6} 5 s^{1}$ & 30 & 5.849 & 4.7 & 24 & -4 & 0.69 & -18 \\
& GGA $^{\mathrm{b}}$ & $4 p^{6} 5 s^{1}$ & 60 & 5.847 & 4.7 & 26 & 4 & 0.70 & -17 \\
\hline \hline
\end{tabular}

${ }^{\mathrm{a} C e p e r l e y-A l d e r-P e r d e w-Z u n g e r ~(R e f s . ~} 23$ and 24).

${ }^{\mathrm{b}}$ Perdew-Burke-Ernzerhof (Ref. 25).

lations, have been corrected for the spin polarization energy (152, 148, and $132 \mathrm{meV}$ for $\mathrm{Al}, \mathrm{K}$, and $\mathrm{Rb}$, respectively). This correction has to be applied because we use a non-spinpolarized code. Since both the Al substrate and the alkali metal on Al substrate systems are spin compensated, the code can be directly applied to them. An isolated alkali atom, however, is spin polarized: According to Hund's rule the spin-compensated state is not the ground state but electrons in a single and not fully occupied atomic orbital try to maximize their spin state. A well-established technique to take this effect into account is to calculate the energy difference between an atom in spin-polarized and spin-compensated states. This energy difference has been calculated for all elements and is listed in Ref. 22. For all total-energy calculations zero-point vibrations have not been included. The energy cutoff has been chosen to give errors in the adsorption energy of less than $2 \mathrm{meV}$. In order to achieve these error bars in the surface energies total energies have been converged up to an accuracy of $10^{-5}$ hartree. The equilibrium structures have been obtained by enforcing that the Hellmann-Feynman forces on all surface atoms be less than $10^{-4}$ hartree/bohr. In order to minimize the slab thickness we use asymmetric slabs where the alkali atoms are adsorbed only on one side of the slab. A consequence of this geometry is that two inequivalent surfaces exist: one with alkali adsorbates and a bare one on the other side of the slab. Since in general the two surfaces have different work functions, the periodic boundary conditions cause an artificial electric field in the vacuum region. ${ }^{3}$ As has been shown in Ref. 3 this field can be efficiently eliminated by applying a dipole correction (by placing a self-consistently calculated dipole layer in the vacuum region). Using this approach a slab thickness of four Al layers was found to be sufficient. Details of the method and the computer programs are described in Refs. 19 and 20. Additional convergence checks have been reported in Ref. 3 .

The calculations have been performed using both the local density approximation ${ }^{23,24}$ (LDA) and the Perdew-BurkeErnzerhof generalized gradient approximation ${ }^{25}$ (PBEGGA). For both exchange- (XC)- energy functionals the appropriate pseudopotentials have been generated. Moreover, to test and analyze the effect of the alkali semicore states on the bonding geometry and energetics we have generated two sets of pseudopotentials for the alkalis: one in which the semicore $p$ states have been explicitly included into the valence shell, the other where they have been kept frozen and treated only in the nonlinear core-valence exchangecorrelation (NLCC) approximation.

\section{B. Definitions}

Here and in the following we will focus on the on-top position when considering on-surface adsorption: The on-top position has been clearly identified in a LEED analysis for $(\sqrt{3} \times \sqrt{3}) R 30 \mathrm{~K}$ and $\mathrm{Rb}$ on $\mathrm{Al}(111)$. The on-top and substitutional adsorption energies have been calculated following Ref. 3:

$$
\begin{aligned}
E_{\mathrm{ad}}^{\text {on-top }} & =-\left(E_{\mathrm{tot}}^{\text {on-top }}-E_{\text {tot }}^{\text {clean }}-E_{\text {tot }}^{\text {free }}\right) \\
E_{\mathrm{ad}}^{\text {subst }} & =-\left(E_{\text {tot }}^{\text {subst }}-\left(E_{\text {tot }}^{\text {clean }}+E_{\text {tot }}^{\text {bulk }}\right)-E_{\text {tot }}^{\text {free }}\right) .
\end{aligned}
$$


TABLE II. The calculated surface geometries and adsorption energies of $\mathrm{Al}(111)-(\sqrt{3} \times \sqrt{3}) R 30: \mathrm{K}$ and $\mathrm{Rb}$ structures in comparison with experimental data reported by Adams (Ref. 13). Here, $E_{\text {cut }}$ is the cutoff energy of the plane-wave basis set, $d_{\text {alkali-Al }}$ the vertical spacing between the adsorbed alkali layer and the first substrate layer, $d_{\mathrm{Al}-\mathrm{Al}}$ the vertical spacing between the first and the second layer of the substrate, $\Delta(r)$ the vertical splitting of the first Al layer, and $E_{\text {ad }}$ the adsorption energy (see Fig. 1)

\begin{tabular}{|c|c|c|c|c|c|c|c|}
\hline Structure & $\begin{array}{l}\text { Data } \\
\text { from }\end{array}$ & $\begin{array}{c}\text { Valence } \\
\text { shell }\end{array}$ & $\begin{array}{l}E_{\text {cut }} \\
(\mathrm{Ry})\end{array}$ & $\begin{array}{c}d_{\text {alkali-Al }} \\
(\AA)\end{array}$ & $\begin{array}{c}d_{\mathrm{Al}-\mathrm{Al}} \\
(\AA)\end{array}$ & $\begin{array}{c}\Delta(r) \\
(\AA)\end{array}$ & $\begin{array}{l}E_{\text {ad }} \\
(\mathrm{eV})\end{array}$ \\
\hline \multirow[t]{3}{*}{ Clean $\mathrm{Al}(111)$} & Experiment & - & - & - & $2.36 \pm 0.01$ & - & - \\
\hline & $\operatorname{LDA}^{\mathrm{a}}$ & $3 s^{2} 3 p^{1}$ & 45 & - & 2.31 & & - \\
\hline & $\mathrm{GGA}^{\mathrm{b}}$ & $3 s^{2} 3 p^{1}$ & 50 & & 2.37 & & \\
\hline \multirow[t]{4}{*}{ K:on-top } & Experiment & - & - & $3.08 \pm 0.03$ & $2.33 \pm 0.02$ & $0.28 \pm 0.02$ & - \\
\hline & $\mathrm{LDA}^{\mathrm{a}}$ & $4 s^{1}$ & 20 & 2.93 & 2.41 & 0.25 & 1.460 \\
\hline & $\operatorname{LDA}^{a}$ & $3 p^{6} 4 s^{1}$ & 45 & 3.13 & 2.37 & 0.19 & 1.298 \\
\hline & GGA $^{b}$ & $3 p^{6} 4 s^{1}$ & 50 & 3.13 & 2.31 & 0.19 & 1.122 \\
\hline \multirow[t]{4}{*}{ K:subst. } & Experiment & - & - & $2.16 \pm 0.02$ & $2.27 \pm 0.02$ & - & - \\
\hline & $\operatorname{LDA}^{\mathrm{a}}$ & $4 s^{1}$ & 20 & 2.15 & 2.23 & - & 1.574 \\
\hline & $\operatorname{LDA}^{a}$ & $3 p^{6} 4 s^{1}$ & 45 & 2.37 & 2.22 & - & 1.339 \\
\hline & $\mathrm{GGA}^{\mathrm{b}}$ & $3 p^{6} 4 s^{1}$ & 50 & 2.59 & 2.26 & - & 1.173 \\
\hline \multirow[t]{4}{*}{ Rb:on-top } & Experiment & - & - & $3.22 \pm 0.03$ & $2.33 \pm 0.02$ & $0.27 \pm 0.02$ & - \\
\hline & $\operatorname{LDA}^{\mathrm{a}}$ & $5 s^{1}$ & 20 & 2.99 & 2.41 & 0.25 & 1.478 \\
\hline & $\mathrm{LDA}^{\mathrm{a}}$ & $4 p^{6} 5 s^{1}$ & 25 & 3.22 & 2.38 & 0.20 & 1.305 \\
\hline & GGA $^{b}$ & $4 p^{6} 5 s^{1}$ & 30 & 3.37 & 2.39 & 0.17 & 1.085 \\
\hline \multirow[t]{4}{*}{ Rb:subst. } & Experiment & - & - & $2.41 \pm 0.02$ & $2.27 \pm 0.02$ & - & - \\
\hline & $\operatorname{LDA}^{\mathrm{a}}$ & $5 s^{1}$ & 20 & 2.28 & 2.22 & - & 1.562 \\
\hline & $\mathrm{LDA}^{\mathrm{a}}$ & $4 p^{6} 5 s^{1}$ & 25 & 2.56 & 2.22 & - & 1.322 \\
\hline & $\mathrm{GGA}^{\mathrm{b}}$ & $4 p^{6} 5 s^{1}$ & 30 & 2.70 & 2.25 & - & 1.103 \\
\hline
\end{tabular}

${ }^{\mathrm{a}}$ Ceperley-Alder-Perdew-Zunger (Refs. 23 and 24).

${ }^{b}$ Perdew-Burke-Ernzerhof (Ref. 25).

Here, the quantities appearing on the right sides are ab initio total energies: $E_{\mathrm{tot}}^{\text {on-top }}$ and $E_{\mathrm{tot}}^{\text {subst }}$ those of the adsorbate systems (on-top and substitutional geometries, respectively), $E_{\text {tot }}^{\text {clean }}$ that of the clean Al (111) slab, and $E_{\text {tot }}^{\text {bulk }}$ that of a substrate bulk atom. $E_{\text {tot }}^{\text {free }}$ is the total energy of the isolated spin-polarized adsorbate atom. In the above equation the substitutional adsorption energy has been defined by assuming that the substituted substrate atom becomes a bulk atom. Physically this may mean, e.g., that the kicked-out substrate atom forms first a mobile adatom on the surface which eventually gets trapped at a kink site at a surface step. Since adsorption at this site reproduces the surface (the only change is that the kink moved along the step edge), the adsorption energy of it is exactly the bulk cohesive energy. ${ }^{3}$

\section{RESULTS AND DISCUSSION}

\section{A. Elementary bulk phases}

We have first tested the various sets of pseudopotentials (LDA and GGA, with and without semicore states) by calculating the cohesive energy, equilibrium lattice constant, and bulk modulus of the elementary bulk phases of $\mathrm{Al}, \mathrm{K}$, and $\mathrm{Rb}$ (bcc structure). Based on the results listed in Table I we can draw the following conclusions. For Al, going from the LDA to the GGA significantly improves all calculated quantities: as expected the GGA corrects for the LDA overbinding, thus reducing the cohesive energy and the bulk modulus and increasing the lattice constant. In fact, in the case of $\mathrm{Al}$ the PBE-GGA performs very well. For the alkalis a slightly different picture emerges. Let us first focus on the LDA results. Here we find that the inclusion of the semicore states (the $3 p$ and $4 p$ electrons for $\mathrm{K}$ and $\mathrm{Rb}$, respectively) significantly improves all calculated properties. The lattice constant and cohesive energy get very close (error $<0.5 \%$ for the lattice constant and $<2 \%$ for the cohesive energy) to the corresponding experimental values. Using the PBE-GGA for exchange correlation again the expected behavior is found: the cohesive energy and bulk modulus decrease and the lattice constant increases. However, in contrast to what has been found for $\mathrm{Al}$ these corrections overcorrect the LDA (with the exception of the bulk modulus)_for bulk alkalis the PBE-GGA performs worse than the LDA with respect to the lattice constant and cohesive energy. These systems might be therefore a good benchmark system for new and improved exchange-correlation functionals. 


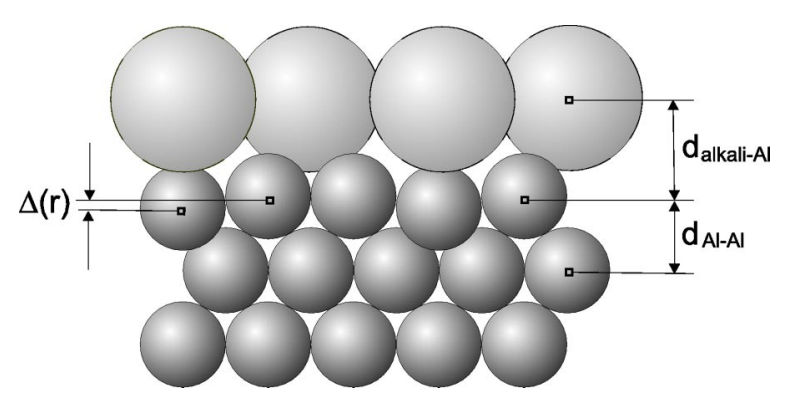

FIG. 1. The $\mathrm{Al}(111)-(\sqrt{3} \times \sqrt{3}) R 30: \mathrm{Rb}(\mathrm{K})$ structure. Schematic side view. The indicated distances correspond to those in Table II.

\section{B. Alkali adsorption on Al (111)}

In a next step we have used different sets of pseudopotentials and exchange-correlation functionals to study the adsorption geometry and energy of $\mathrm{K}$ and $\mathrm{Rb}$ in the ordered $(\sqrt{3} \times \sqrt{3}) R 30$ phase on $\mathrm{Al}(111)$. Both on-top and substitutional adsorption have been considered. The results are listed in Table II (see also Fig. 1). Let us first focus on trends for the different functionals and pseudopotentials. Including the semicore states into the valence prevents the alkali atom from getting too close to the Al surface. Thus, in all cases (for $\mathrm{K}$ and $\mathrm{Rb}$ and for on-top and substitutional sites) the alkali-aluminum bond length increases and the adsorption energy decreases. The fact that including the semicore states reduces the adsorbate-substrate interaction can be also seen in the Al-Al spacing between the first and second layers and the rumpling $\Delta r$ of the first substrate layer: both get closer to the situation without alkali adsorption. It is interesting to note that previous studies performed for qualitatively different substrates (alkali adsorbates on transition metal surfaces) observed a very similar effect ${ }^{26,27}$ : The semicore states were found to lead, e.g., to a significant counterpolarization of the alkali adatoms and to affect the adsorbate-substrate bonds. The PBE-GGA shows the expected behavior-it increases the alkali-adsorbate bond length and decreases the adsorption energy.

A comparison with the experimentally derived structural parameters shows generally good agreement for all cases with error bars $<0.1 \AA$ except for the alkali-aluminum bond length. The latter depends sensitively on the choice of functional and the treatment of the semicore states. The LDA and inclusion of the semicore states works well for the on-top configuration but slightly overestimates the bond length in the substitutional configuration.

The GGA generally overestimates the alkali-aluminum bond length. While GGA functionals are well known to overestimate bond lengths the discrepancy found in the case of the substitutional site is unusually large: It is $\approx 0.3 \AA$ for $\mathrm{Rb}$ and $\approx 0.4 \AA$ for $K$ (see Table II). From our results we cannot unambiguously identify the source of the discrepancy: One reason might be the (still insufficient) representation of the alkali atomic core by keeping the remaining core states frozen (see, e.g., Ref. 28). This would, e.g., explain why the error for the substitutional site is larger than for the on-top configuration: In the substitutional configuration the bonding is known to be more covalent (in comparison with the on-top
TABLE III. Comparison of the energy differences between alkali adsorption on on-top and substitutional sites.

\begin{tabular}{lcccc}
\hline \hline Element & $\begin{array}{c}\text { Valence } \\
\text { shell }\end{array}$ & $\begin{array}{c}\text { XC } \\
\text { functional }\end{array}$ & $\begin{array}{c}E_{\text {cut }} \\
(\mathrm{Ry})\end{array}$ & $\begin{array}{c}E_{\text {ad }}^{\text {subst }}-E_{\text {ad }}^{\text {on-top }} \\
(\mathrm{meV})\end{array}$ \\
\hline $\mathrm{Na}^{\mathrm{a}}$ & $3 s^{1}$ & LDA & 12 & 300 \\
$\mathrm{~K}$ & $4 s^{1}$ & LDA & 20 & 114 \\
$\mathrm{~K}$ & $3 p^{6} 4 s^{1}$ & LDA & 45 & 41 \\
$\mathrm{~K}$ & $3 p^{6} 4 s^{1}$ & PBE-GGA & 50 & 51 \\
$\mathrm{Rb}$ & $5 s^{1}$ & LDA & 20 & 84 \\
$\mathrm{Rb}$ & $4 p^{6} 5 s^{1}$ & LDA & 25 & 17 \\
$\mathrm{Rb}$ & $4 p^{6} 5 s^{1}$ & PBE-GGA & 30 & 19 \\
\hline \hline
\end{tabular}

${ }^{a}$ Neugebauer and Scheffler (Ref. 3).

site) and core polarization effects may be more important. Another reason might be the choice of exchange-correlation potential. To unambiguously distinguish between the two effects (core electrons, XC potential) all-electron calculations using different $\mathrm{XC}$ potentials are needed.

\section{Comparison between on-top on substitutional adsorption}

By looking at the energy difference between on-top and substitutional site $\left(\Delta E=E_{\mathrm{ad}}^{\text {subst }}-E_{\mathrm{ad}}^{\text {on-top }}\right)$ we can identify the energetically stable site-a positive value indicates that the substitutional site is more stable than the on-top configuration. The energy differences are listed in Table III. In all cases the substitutional site is more stable than the on-top configuration. Looking at the numbers a clear chemical trend can be observed: Going down the periodic table (i.e., from $\mathrm{Na}$ to $\mathrm{K}$ to $\mathrm{Rb}$ ) the preference for the substitutional site decreases but even for $\mathrm{Rb}$ it is the most stable configuration. The numbers also clearly show that the semicore states have a significant affect on the alkali adsorption: By reducing the adsorbate-substrate interaction they also strongly reduce the preference for the substitutional site. Finally, we note that in contrast to the structural parameters and the absolute adsorption energies the LDA- and GGA-calculated energy differences are very close (energy difference $<10 \mathrm{meV}$ ).

\section{CONCLUSIONS}

We have studied on-top and substitutional adsorption of $\mathrm{K}$ and $\mathrm{Rb}$ alkali adsorbates on the $\mathrm{Al}(111)-(\sqrt{3} \times \sqrt{3}) R 30$ surface. A specific focus of this work was to identify the role of the alkali semicore states on the bonding geometry and energy and the performance of various exchange-correlation functionals (LDA vs PBE-GGA). Our results showed that independent of these choices the substitutional site is energetically more favorable than the on-top geometry. Also, clear quantitative changes and trends could be observed. Inclusion of the alkali semicore states in the valence was found to be crucial otherwise, the alkali substrate interaction is overestimated, resulting in too small alkali-substrate bond 
lengths and too high adsorption energies. Furthermore, the PBE-GGA generally overestimates the alkali-aluminum bond length compared to experiment (by up to $0.3 \AA$ ). The fact that this system is one of the simplest chemisorption systems and that accurate structural parameters exist makes it an interesting benchmark to test the performance of new and optimized exchange-correlation functionals.

\section{ACKNOWLEDGMENTS}

One of the authors (P.S.) would like to thank A. Kiejna and J. Peisert for their assistance and help in the course of the project and also M. Fuchs and C. Stampfl for useful discussions. The work has been supported by the FritzHaber-Institut der Max-Planck-Gesellschaft, Germany.
*On leave from Wroclaw University of Technology, Wyb. Wyspianskiego 27, 50-370 Wroclaw, Poland. Electronic address: Pawel.Scharoch@pwr.wroc.pl

${ }^{1}$ A. Schmalz, S. Aminpirooz, L. Becker, J. Haase, J. Neugebauer, and M. Scheffler, Phys. Rev. Lett. 67, 2163 (1991).

${ }^{2}$ C. Stampfl, M. Scheffler, H. Over, J. Burchhardt, M. Nielsen, D.L. Adams, and W. Moritz, Phys. Rev. Lett. 69, 1532 (1992).

${ }^{3}$ J. Neugebauer and M. Scheffler, Phys. Rev. B 46, 16067 (1992).

${ }^{4}$ J.N. Andersen, E. Lundgren, R. Nyholm, and M. Qvarford, Phys. Rev. B 46, 12784 (1992).

${ }^{5}$ J.N. Andersen, E. Lundgren, R. Nyholm, and M. Qvarford, Surf. Sci. 289, 307 (1993).

${ }^{6}$ C. Stampfl, M. Scheffler, H. Over, J. Burchhardt, M. Nielsen, and D.L. Adams, Phys. Rev. B 49, 4959 (1994).

${ }^{7}$ J. Burchhardt, M.M. Nielsen, and D.L. Adams, Phys. Rev. B 50, 4718 (1994).

${ }^{8}$ M.M. Nielsen, J. Burchhardt, and D.L. Adams, Phys. Rev. Lett. 72, 3370 (1994).

${ }^{9}$ C. Stampfl, J. Neugebauer, and M. Scheffler, Surf. Sci. 307-309, 8 (1994).

${ }^{10}$ J. Wang, Z.C. Ying, and E.W. Plummer, Phys. Rev. B 51, 5590 (1995)

${ }^{11}$ E. Lundgren, R. Nyholm, J. Burchhardt, D. Heskett, and J.N. Andersen, Surf. Sci. 343, 37 (1995).
${ }^{12}$ M.M. Nielsen, J. Burchhardt, D.L. Adams, and J.N. Andersen, Phys. Rev. B 58, 12655 (1998).

${ }^{13}$ D.L. Adams, Appl. Phys. A 62, 123 (1996).

${ }^{14}$ B. Wenzien, J. Bormet, J. Neugebauer, and M. Scheffler, Surf. Sci. 287/288, 559 (1993).

${ }^{15}$ J. Neugebauer and M. Scheffler, Phys. Rev. Lett. 71, 557 (1993).

${ }^{16}$ R.J. Needs and G. Rajagopal, Surf. Sci. 372, 179 (1997).

${ }^{17}$ J.B. Taylor and I. Langmuir, Phys. Rev. 44, 423 (1933).

${ }^{18}$ R.W. Gurney, Phys. Rev. 47, 479 (1935).

${ }^{19}$ M. Bockstedte, A. Kley, J. Neugebauer, and M. Scheffler, Comput. Phys. Commun. 107, 187 (1997).

${ }^{20}$ M. Fuchs and M. Scheffler, Comput. Phys. Commun. 119, 67 (1999).

${ }^{21}$ N. Troullier and J.L. Martins, Phys. Rev. B 43, 1993 (1991).

${ }^{22}$ http://physics.nist.gov/PhysRefData/DFTdata/.

${ }^{23}$ D.M. Ceperley and B.J. Alder, Phys. Rev. Lett. 45, 567 (1980).

${ }^{24}$ J.P. Perdew and A. Zunger, Phys. Rev. B 23, 5048 (1981).

${ }^{25}$ J.P. Perdew, K. Burke, and M. Ernzerhof, Phys. Rev. Lett. 77, 3865 (1996).

${ }^{26}$ E. Wimmer, A.J. Freeman, J.R. Hiskes, and A.M. Karo, Phys. Rev. B 28, 3074 (1983).

${ }^{27}$ S.R. Chubb, E. Wimmer, A.J. Freeman, J.R. Hiskes, and A.M. Karo, Phys. Rev. B 36, 4112 (1987).

${ }^{28}$ M. Rohlfing, P. Kruger, and J. Pollmann, Phys. Rev. Lett. 57, 6485 (1998). 